\title{
Diversity among satellite glial cells in dorsal root ganglia of the rat
}

\author{
R.S. Nascimento ${ }^{1,2}$, M.F. Santiago ${ }^{3}$, S.A. Marques ${ }^{1,2}$, S. Allodi ${ }^{1,2}$ and A.M.B. Martinez ${ }^{1,2}$ \\ ${ }^{1}$ Departamento de Histologia e Embriologia, ${ }^{2}$ Programa de Pós-Graduação em Ciências Morfológicas, \\ Instituto de Ciências Biomédicas, ${ }^{3}$ Instituto de Biofísica Carlos Chagas Filho, Centro de Ciências da \\ Saúde, Universidade Federal do Rio de Janeiro, Rio de Janeiro, RJ, Brasil \\ Correspondence to: A.M.B. Martinez, Departamento de Histologia e Embriologia, ICB, CCS, Bloco F, \\ UFRJ, Av. Brig. Trompowsky, s/n, 21941-540 Rio de Janeiro, RJ, Brasil \\ E-mail: martinez@histo.ufrj.br
}

\begin{abstract}
Peripheral glial cells consist of satellite, enteric glial, and Schwann cells. In dorsal root ganglia, besides pseudo-unipolar neurons, myelinated and nonmyelinated fibers, macrophages, and fibroblasts, satellite cells also constitute the resident components. Information on satellite cells is not abundant; however, they appear to provide mechanical and metabolic support for neurons by forming an envelope surrounding their cell bodies. Although there is a heterogeneous population of neurons in the dorsal root ganglia, satellite cells have been described to be a homogeneous group of perineuronal cells. Our objective was to characterize the ultrastructure, immunohistochemistry, and histochemistry of the satellite cells of the dorsal root ganglia of 17 adult 3-4-month-old Wistar rats of both genders. Ultrastructurally, the nuclei of some satellite cells are heterochromatic, whereas others are euchromatic, which may result from different amounts of nuclear activity. We observed positive immunoreactivity for S-100 and vimentin in the cytoplasm of satellite cells. The intensity of S-100 protein varied according to the size of the enveloped neuron. We also noted that vimentin expression assumed a ring-like pattern and was preferentially located in the cytoplasm around the areas stained for S-100. In addition, we observed nitric oxide synthase-positive small-sized neurons and negative large-sized neurons equal to that described in the literature. Satellite cells were also positive for NADPH-diaphorase, particularly those associated with small-sized neurons. We conclude that all satellite cells are not identical as previously thought because they have different patterns of glial marker expression and these differences may be correlated with the size and function of the neuron they envelope.
\end{abstract}

Key words: Satellite glial cells; Peripheral nervous system; Vimentin; S-100; Electron microscopy

Research supported by CNPq (\#473063/2007-7) and FAPERJ (\#E-26/152.877/2006).

Received March 20, 2008. Accepted August 18, 2008

In recent decades, glial cells have attracted the attention of neuroscientists because of the important roles attributed to them. It is widely accepted that the proper functioning of neurons depends on the support of glial cells (1). Glial cells are mainly classified according to their morphological aspect or their location, which may be central or peripheral (2). The peripheral glia consist of satellite glial cells (SGC), enteric glial cells (EGC), and Schwann cells (SC). The majority of studies on peripheral glia are restricted to SC, perhaps because of their plasticity and importance for nerve degeneration and regeneration (3). There are few reports concerning SGC and EGC, some of which have even confounded SGC with SC and EGC. In a review, Hanani (4) reported that SGC have been neglected over the years, and that most reports on sensory ganglia are focused on the neurons and not on their ensheathing cells.

The dorsal root ganglia (DRG) are composed of three classes of neurons classified according to morphology and function $(5,6)$. DRG glial cells include SGC and SC. The morphology of SGC was carefully studied by Pannese (5) and few investigators have attempted to further characterize them. Vega et al. (7) suggested that there is a heterogeneous population of SGC, based on the expression of 
different cytoskeleton proteins. More recently, Hanani (4) focused on SGC and suggested that because they share many characteristics with the central nervous system glia, they may participate in the neuronal signaling process and transmission.

Because SGC may be crucial for pathological degeneration and for regeneration of sensory ganglion neurons, and because specific literature on their structure and function is not abundant, the aim of the present study was to further characterize the SGC in DRG by transmission electron microscopy (TEM), immunohistochemistry (IHC) using specific markers for glial cells, such as S-100 (A and $B$, which may also label some neurons) and vimentin, and for neuronal nitric oxide (NO) synthase, and also by histochemistry $(\mathrm{HC})$ for NADPH-diaphorase. Additionally, the relationship between SGC and neurons was taken into consideration. Our observations showed that there are different populations of SGC, and that they may be related to the different types of neurons.

In this study, adult 3-4-month-old Wistar rats of both genders ( 5 for TEM, 9 for $\mathrm{IHC}$, and 3 for $\mathrm{HC}$ ) were used. This research was approved by the Commission of Animal Care of the Instituto de Biofísica, Universidade Federal do Rio de Janeiro. The rats were anesthetized with intraperitoneal sodium pentobarbital ( $30 \mathrm{mg} / \mathrm{kg}$ ) and perfused transcardially with fixative solution containing $2 \%$ glutaraldehyde and $4 \%$ paraformaldehyde. After perfusion, the L4 and L5 DRG of both sides of 5 animals destined for TEM were removed and immersed overnight in $2.5 \%$ glutaraldehyde in $0.1 \mathrm{M}$ phosphate buffer, washed in phosphate buffer and then in $0.1 \mathrm{M}$ cacodylate buffer (CB), and postfixed in $1 \%$ osmium tetroxide containing $0.8 \%$ potassium ferrocyanide and $5 \mathrm{mM}$ calcium chloride in CB. The specimens were then washed in $\mathrm{CB}$, block-stained with $1 \%$ uranyl acetate and then dehydrated in a graded acetone series, infiltrated and embedded in Poly/Bed 812 resin (Polysciences, Inc., USA), and polymerized at $60^{\circ} \mathrm{C}$ for 48 h. Five hundred-nanometer semithin sections were stained with toluidine blue and used for light microscopic studies. Ultrathin sections $(60-70 \mathrm{~nm})$ were collected on copper grids, and stained with 5\% uranyl acetate and Reynold's lead citrate. TEM studies were carried out using a Zeiss 900 transmission electron microscope (Germany) operated at $80 \mathrm{kV}$.

After dissection and post-fixation for $4 \mathrm{~h}$ in fresh fixative solution ( $2 \%$ glutaraldehyde and $4 \%$ paraformaldehyde), the DRG of 9 animals were cryoprotected in $30 \%$ sucrose, and embedded ice-cold in Tissue Tek $^{\circledR}$ O.C.T. ${ }^{\mathrm{M}}{ }^{\mathrm{M}}$ compound (Sakura Finetek Europe B.V., the Netherlands). Ten-micrometer thick sections were obtained with a cryostat (Leica CM 1850, Germany), collected on gelatin-coated glass slides, washed three times in phosphate-buffered saline (PBS) with $0.3 \% \mathrm{X}-100$ Triton. The sections were then washed in the previous solution to which normal goat serum $(10 \%, v / v)$ was added and incubated with primary antibody [glial fibrillary acidic protein (GFAP), 1:100, Novocastra, UK; S-100, 1:100, Sigma, USA; vimentin, 1:100, Dako, Denmark; neuronal NO synthase, 1:200, Sigma] overnight. After rinsing, they were incubated with the secondary antibody for $2 \mathrm{~h}$ [fluorescein isothiocyanate (FITC) or tetramethylrhodamine isothiocyanate (TRITC) at a dilution of 1:100], rinsed again and mounted with buffered glycerol mounting medium containing $1 \% \mathrm{n}$-propylgallate (Sigma). Primary antibodies were omitted for negative controls. DAPI nuclear staining (1:2000, Sigma) was used for some samples. The IHC for NO synthase was revealed using DAB peroxidase substrate (Sigma) and the sections were counter-stained with hematoxylin. Sections were observed and photographed under a Zeiss axiophot fluorescence microscope or a Zeiss confocal microscope LSM 510 Meta.

The sections, obtained as described above with a cryostat, and collected on gelatin-coated glass slides, were washed three times in $0.1 \mathrm{M} \mathrm{PBS}, \mathrm{pH} 7.4$, plus $0.05 \%$ Tween and immersed in the following solution: $1 \mathrm{mM}$ ß$\mathrm{NADPH}$ reduced form, and $0.5 \mathrm{mM}$ nitroblue tetrazolium dissolved in Tris/ $\mathrm{HCl}$ buffer $(0.05 \mathrm{M}, \mathrm{pH} 7.5)$. The reaction was performed with constant stirring for $1-4 \mathrm{~h}$ at $37^{\circ} \mathrm{C}$. The sections were washed in $\mathrm{Tris} / \mathrm{HCl}$ buffer, dehydrated in alcohol, cleared in xylene, coverslipped with Entellan (Merck, Germany), and analyzed and photographed using a Zeiss axioscope light microscope.

Figure 1A shows a light microscopic image of the DRG, illustrating neurons and satellite glial cells. Figure $1 B, C$ reveal the relationship between neurons and SGC as seen at the ultrastructural level. We observed some neurons with electron-dense cytoplasm, others with electron-lucent cytoplasm, and still others with intermediary electron densities (Figure 1B). Neuron cell bodies were completely ensheathed by the SGC. Sometimes only a thin ring of cytoplasm was observed around the soma of the neuron (Figure 1C). The close apposition of the membranes of the two cells is important because it shows that the SGCs may be a route for communication between the neurons and the extra-cellular environment. Sometimes a neuronal projection was seen invading the cytoplasm of the SGC (Figure $1 \mathrm{~B}$ and inset).

Figure 1D-F shows the ultrastructure of SGC and their ensheathing neurons. Clearly, different electron densities of the nuclei and cytoplasm of the SGC were observed, which may be correlated with different types of neurons. Thus, SGC showing heterochromatic nuclei and electron- 

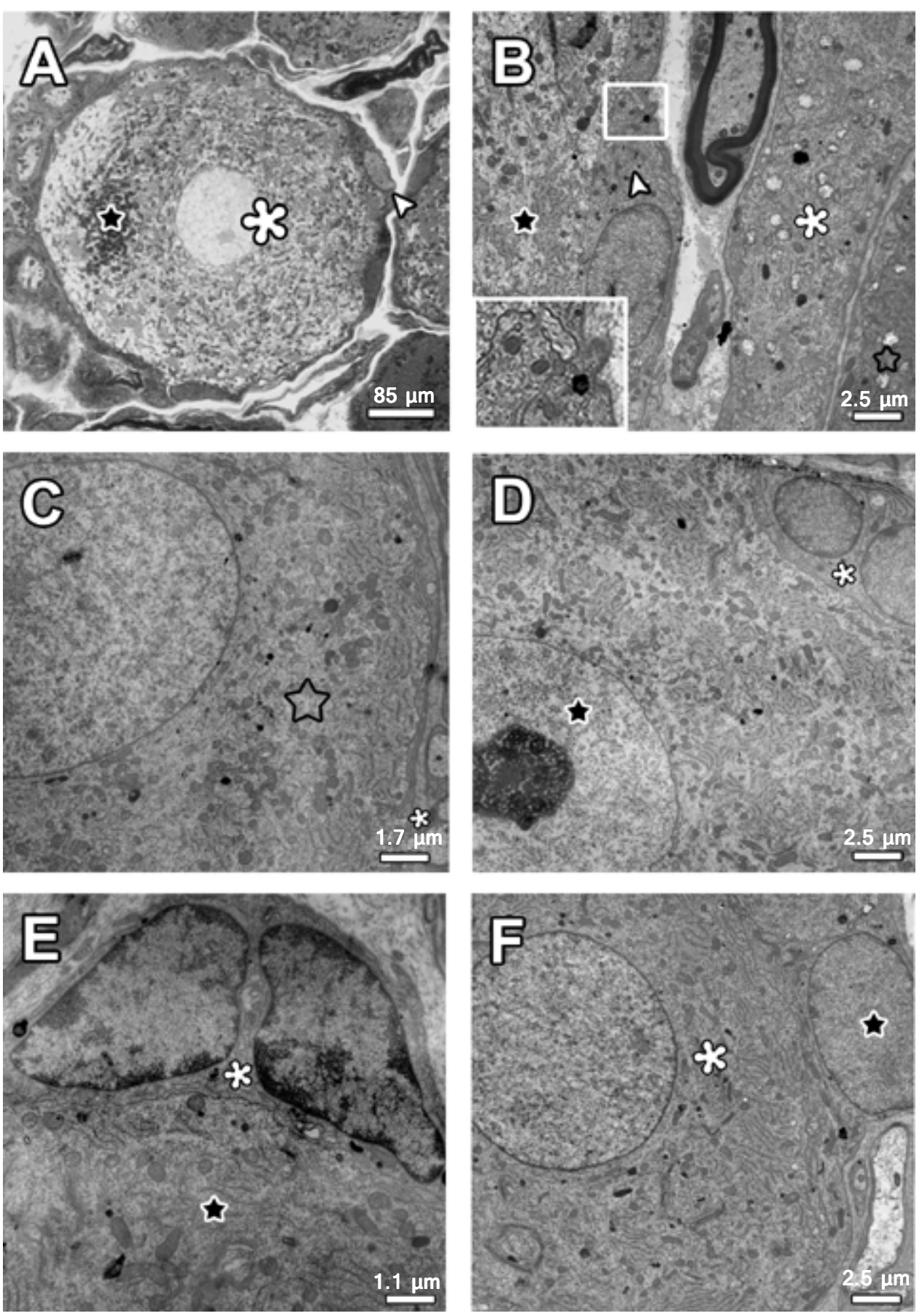

Figure 1. Light micrograph. $A$, Semithin section of dorsal root ganglion showing the relationship between a large neuron (asterisk) and satellite glial cells. The nuclei of a satellite glial cell is visible (arrowhead). The black star indicates an accumulation of Nissl substance. $B$, Electron micrograph of dorsal root ganglion. Note electron-lucent neuron (black star), electron-dense neuron (open star), and an intermediate neuron (asterisk). The arrowhead indicates a satellite glial cell. The boxed area includes a fine process projecting into invaginations of the adjacent satellite glial cell (enlarged in the inset). $C$, A dorsal root ganglion neuron (open star) at a higher magnification showing the surrounding thin satellite glial cell cytoplasm (asterisk). $D$, Large-light neuron (star) and its surrounding satellite glial cell with two nuclei (asterisk). E, Large electron-lucent neuron (black star) and the adjacent satellite glial cell (asterisk) showing heterochromatic nuclei. F, Small dark neuron (asterisk) and an adjacent satellite glial cell (black star) with an electron-lucent nucleus. Magnification bar: A: $85 \mu \mathrm{m}$; B, D, F: $2.5 \mu \mathrm{m}$; C: $1.7 \mu \mathrm{m}$, E: $1.1 \mu \mathrm{m}$. 
dense cytoplasm were preferentially contacting neurons with electron-lucent cytoplasm, whereas those with euchromatic nuclei and electron-lucent cytoplasm were contacting neurons with electron-dense cytoplasm (Figure 1D-F).

IHC for S-100 protein disclosed a widely varying pattern of immunolabeling for the DRG neurons: small-diameter neurons lacked immunoreactivity, whereas large-sized neurons displayed intense labeling (Figure 2A). Interestingly, the glial cell population surrounding large neurons exhibited intense immunoreactivity, whereas those surrounding small neurons were weakly labeled. The reaction product in SGC was preferentially localized around the nuclei. A positive reaction could also be observed in the cytoplasm of SC, particularly in the perinuclear region (Figure 2B). Areas corresponding to Schmidt-Lantermann incisures were also labeled for S-100 protein. IHC for vimentin protein showed that all SGC were positive for this protein, whereas positivity for vimentin was not seen in neurons. The location of the vimentin label was clearly cytoplasmic, being more evident close to the external membrane.

In order to determine whether the population of SGC stained for S-100 was the same that displayed immunoreactivity for vimentin, we double-labeled both proteins (Figure 2C-F). Interestingly, we did not find cells labeled for just one protein; however, we observed a different preferential location for each protein in the same cells. The S-100 protein was more diffusely distributed throughout the cytoplasm, whereas vimentin was clearly more restricted to the area close to the cytoplasmic membrane (Figure 2C-E). Confocal orthogonal sections confirmed these observations, and clearly showed vimentin concentrated in the vicinity of the SGC external membrane (Figure 2F).

For further characterization of the SGC and the surrounded neurons, we performed a histochemical reaction for NADPH-diaphorase, which revealed the distribution of NO synthase (Figure 2G). Our results showed that large neurons were not as heavily stained as small ones, which displayed a large amount of reaction product. Interestingly, SGC surrounding large neurons were stained for NADPH-diaphorase as well as SGC around heavily stained small neurons. The IHC for neuronal NO synthase confirms our results for NADPH-diaphorase (Figure 2G, inset).

The morphology of the SGC was described extensively by Pannese et al. $(5,8)$. However, since his pioneer work, little has been done to further characterize these cells. In an extensive review, Hanani (4) reported that the SGC have been neglected over the years, and therefore their functions remain unclear. Considering that DRG are composed of morphofunctionally distinct neurons, and also that neurons and SGC have a close anatomical relationship, it is very unlikely that all SGC are similar.

Our results with TEM confirm previous reports describing three different types of neurons; each neuron is completely enveloped by SGC, forming an anatomical unit (5). According to our observations, the neurons classified as large-light are surrounded by SGC showing heterochromatic nuclei, sometimes appearing as double nuclei. Smalldark neurons are surrounded by SGC with euchromatic nuclei. In a recent report, Siemionow et al. (9) studied the ultrastructure of SGC and, based solely on morphological criteria, concluded that there are three different types of SGC. They described two types of SGC with similar morphology to the SGC described here, but they also described a third type, which, according to them, is also a SGC, even though this cell does not come into direct contact with the adjacent neuron. The classification of the third type as a SGC is contrary to the accepted definition of SGC as being in intimate contact with the neuron they ensheath. However, our observations are in agreement with those of Siemionow et al. (9) in terms of the existence of different morphological types of SGC, although we did not attempt to classify these cells. More importantly, our conclusions are not based solely on morphological criteria, but also on the type of proteins that they express. We also found differences related to the type of neurons and the particular set of ensheathing SGC.

The expression of cytoskeletal proteins in SGC was also studied in the present investigation. With respect to S100 , the first evidence that this protein exists outside the central nervous system was provided by Stefansson et al. (10), and later confirmed by González-Martínez et al. (11), who also observed this protein in SGC of DRG, in sympathetic and enteric ganglia. Aquino et al. (12) suggested that the presence of S-100 in SGC is related to the maturity of these cells. Our data for S-100 immunoreactivity in neurons indicated different patterns of expression according to the size of the neuron, which confirm other reports $(10,13,14)$. In SGC, the S-100 immunoreactivity is also quite heterogeneous, and, interestingly, it varies in intensity according to the size of the neurons.

As far as we know, this is the first report that describes different SGC immunolabeling for different types of neurons. We believe that these results are additional clues indicating the presence of different types of SGC in DRG. In relation to the function of the $S-100$ proteins in respect to the neurons and SGC, it is known that $\mathrm{S}-100$ is a calciumbinding protein that may function by buffering of intracellular calcium levels (15), therefore being associated with metabolic activities such as the triggering or activation of proteins inside the cells. Large neurons are known to be 

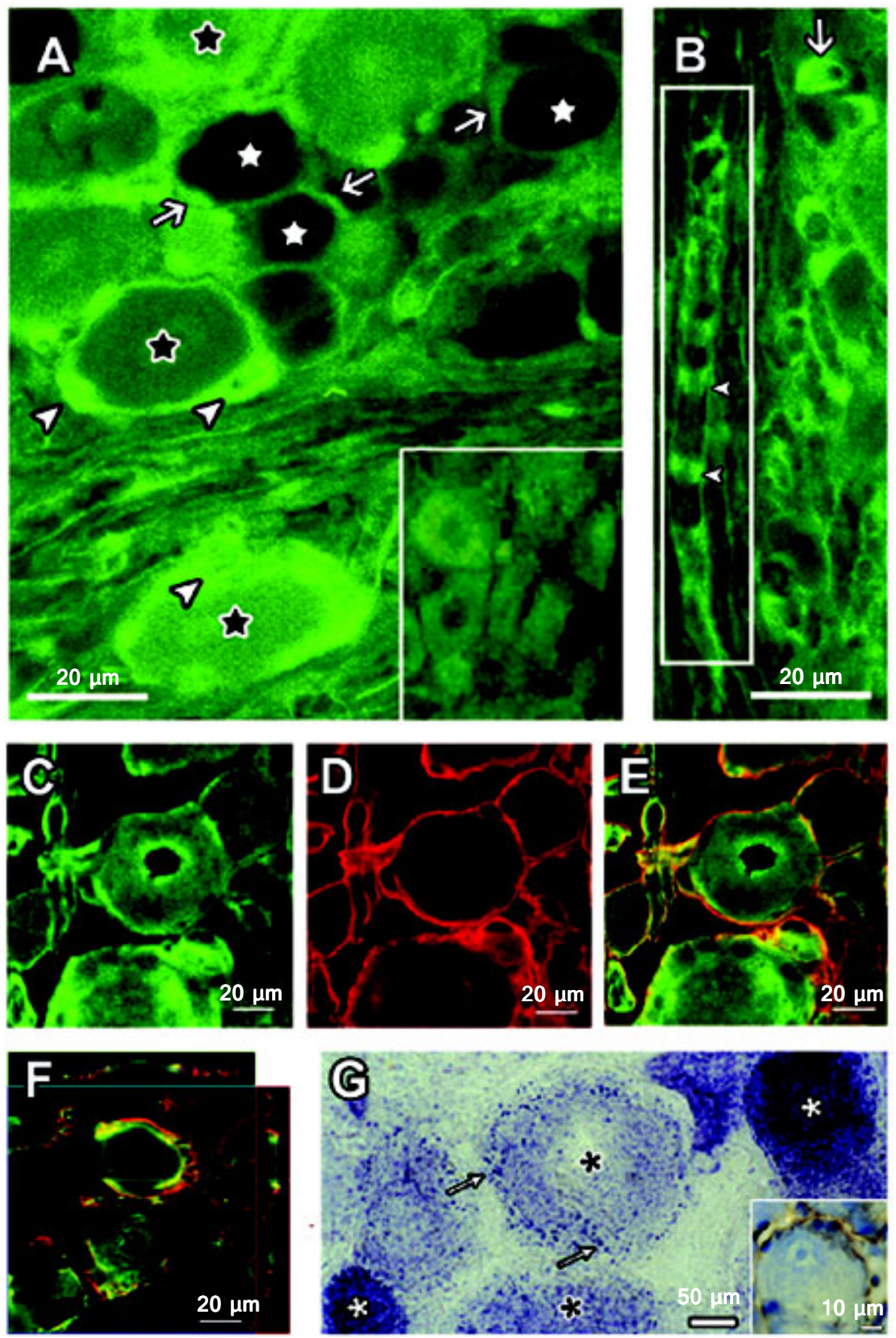

Figure 2. Light micrographs showing the distribution of S-100 (A and B isoforms) in dorsal root ganglia. $A$, Both large neurons (black stars) and their surrounding satellite glial cells (arrowheads) are immunoreactive for S-100 protein. Small-dark neurons (white stars) do not show immunoreactivity, whereas its satellite glial cells (arrows) are lightly stained. The boxed area shows the control reaction. $B$, Pattern of distribution of S-100 protein in a nerve fiber exhibiting the semi-lunar-shaped immunoreaction (arrow) corresponding to the soma of Schwann cells. Inside the boxed area, Schmidt-Lanterman incisures immunoreactive for S-100 protein (arrowheads) are demonstrable. $C-E$, Double immunofluorescence for S-100 and vimentin. Normal pattern of S-100 (C) and vimentin (D) expression. (E) Superposition of $C$ and D. F, Confocal image of S-100 and vimentin expression showing the precise location of the two proteins in the satellite glial cells. $G$, The distribution of NADPH-diaphorase. Note the NADPH-diaphorase-negative large light neurons (black asterisks) surrounded by positive satellite glial cells (arrows). Small dark neurons (white asterisks) and their satellite glial cells display a strong reaction. Inset: Immunohistochemical reaction for neuronal NO synthase. Magnification bar: A-F: $20 \mu \mathrm{m}$; G: $50 \mu \mathrm{m}$, Inset: $10 \mu \mathrm{m}$. 
proprioceptors, and therefore the positive immunoreactivity for S-100 may be related to the function that the neuron and its ensheathing SGC perform in DRG.

Our results for the vimentin immunoreactivity showed that all neurons were negative with respect to this protein, whereas all SGC were positive. This result is partially similar to previous reports (7). According to Vega et al. (7), not all SGC are positive for vimentin; however, we found positive immunoreactivity in the entire population of SGC regardless of the type or size of the neuron. Observation of this reaction by confocal microscopy revealed that the expression of vimentin is more evident near the membrane, which is interesting because it is known that vimentin is a protein that participates in the communication between the cell surface and the nucleus (16). In view of these considerations, our results suggest that vimentin may exert its function through interaction with actin microfilaments, which are typically located under the cytoplasmic membrane. The vimentin reaction in SGC is enhanced after nerve trauma, particularly in those cells located around neurons, which are negative for the antiapoptotic protein $\mathrm{Bcl}-2$. This suggests that the increase in vimentin expression is correlated with neuronal death by apoptosis (17).

The immunoreactivity for GFAP was observed in both SGC and SC, giving a network appearance to the DRG cross-section. No GFAP-positive neurons were found in our sections. The GFAP-immunoreaction product was uniform and diffusely distributed throughout the cytoplasm (data not shown). GFAP is said to abound in the EGC, but its existence in the SGC is controversial. Vega et al. (7) reported that none of the SGC expressed GFAP, whereas other authors reported that SGC express low levels of this protein (4). Our results revealed GFAP positivity in all SGC, supporting recent reports in which GFAP was identified as a marker for peripheral glia (including SGC) differentiation (12).

Functional studies of SGC are even scarcer in the literature. In an attempt to contribute to this field, we performed a cytochemical NADPH-diaphorase reaction to determine whether these cells act as tissue guardians by producing NO. Our results showed that small-dark neurons showed an intense reaction, whereas large-light neurons showed a slight reaction, especially close to the membrane. The SGC around these large neurons were also clearly NADPH-diaphorase-positive, matching the results obtained by Thippeswamy and Morris (18). The SGC around intensely stained small-dark neurons were also positive for NADPH-diaphorase. It is known that small neurons in DRG are those responsible for mediating nociceptive information. Thippeswamy and Morris (18) suggested that these small dark-stained neurons are nociceptive. Thus, the finding of NO production in small-dark neurons reinforces the identification of different types of SGC. Additionally, Aoki et al. (19) observed that the SGC accumulate arginine, which is an NO precursor. Taken together, all these observations suggest that SGC are also producers of NO, since they have all the enzymes necessary for that function. Also, recent data provide evidence that NO has a neuroprotective action, preventing neuronal loss in axotomized or nerve growth factor-deprived DRG neurons (20). In addition, under the same conditions as above, SGC increase the production of CGMP and neurons increase the production of NO (18). Therefore, it is possible to speculate that the NO-protective effect is mediated by the collaboration between the neurons and SGC, as previously suggested (19).

In conclusion, our results strongly indicate that the population of SGC in DRG is morphologically and functionally heterogeneous, and cannot be considered to be a homogeneous group of cells involved in the same supportive role for different types of neurons. Further studies using other approaches, such as cell culture of isolated SGC, are necessary to better clarify the differences among these cells.

\section{References}

1. Fields RD, Stevens-Graham B. New insights into neuronglia communication. Science 2002; 298: 556-562.

2. Reichenbach A. Attempt to classify glial cells by means of their process specialization using the rabbit retinal Muller cell as an example of cytotopographic specialization of glial cells. Glia 1989; 2: 250-259.

3. Jessen KR. Glial cells. Int J Biochem Cell Biol 2004; 36: 1861-1867.

4. Hanani M. Satellite glial cells in sensory ganglia: from form

to function. Brain Res Brain Res Rev 2005; 48: 457-476.

5. Pannese E. The satellite cells of the sensory ganglia. Adv Anat Embryol Cell Biol 1981; 65: 1-111.

6. Sommer EW, Kazimierczak J, Droz B. Neuronal subpopulations in the dorsal root ganglion of the mouse as characterized by combination of ultrastructural and cytochemical features. Brain Res 1985; 346: 310-326.

7. Vega JA, Rodriguez $C$, Medina M, del Valle-Soto ME, Hernandez LC. Expression of cytoskeletal proteins in glial 
cells of dorsal root ganglia. Cell Mol Biol 1989; 35: 635-641.

8. Pannese E, Bianchi R, Calligaris B, Ventura R, Weibel ER. Quantitative relationships between nerve and satellite cells in spinal ganglia. An electron microscopical study. I. Mammals. Brain Res 1972; 46: 215-234.

9. Siemionow K, Weinstein JN, McLain RF. Support and satellite cells within the rabbit dorsal root ganglion: ultrastructure of a perineuronal support cell. Spine 2006; 31: 1882-1887.

10. Stefansson K, Wollmann RL, Moore BW. Distribution of S100 protein outside the central nervous system. Brain Res 1982; 234: 309-317.

11. Gonzalez-Martinez T, Perez-Pinera P, Diaz-Esnal B, Vega JA. S-100 proteins in the human peripheral nervous system. Microsc Res Tech 2003; 60: 633-638.

12. Aquino JB, Hjerling-Leffler $\mathrm{J}$, Koltzenburg $\mathrm{M}$, Edlund $\mathrm{T}$, Villar MJ, Ernfors P. In vitro and in vivo differentiation of boundary cap neural crest stem cells into mature Schwann cells. Exp Neurol 2006; 198: 438-449.

13. Ichikawa H, Jacobowitz DM, Sugimoto T. $S 100$ proteinimmunoreactive primary sensory neurons in the trigeminal and dorsal root ganglia of the rat. Brain Res 1997; 748: 253257.

14. Albuerne M, Mammola CL, Naves FJ, Levanti B, Germana $\mathrm{G}$, Vega JA. Immunohistochemical localization of S100 pro- teins in dorsal root, sympathetic and enteric ganglia of several mammalian species, including man. J Peripher Nerv Syst 1998; 3: 243-253.

15. Donato R. S100: a multigenic family of calcium-modulated proteins of the EF-hand type with intracellular and extracellular functional roles. Int J Biochem Cell Biol 2001; 33: 637668.

16. Sugimura $K$, Haimoto $H$, Nagura $H$, Kato $K$, Takahashi $A$. Immunohistochemical differential distribution of S-100 alpha and S-100 beta in the peripheral nervous system of the rat. Muscle Nerve 1989; 12: 929-935.

17. Raginov IS, Chelyshev YA, Shagidullin TF. Interaction of sensory neurons and satellite cells during stimulation of nerve regeneration. Neurosci Behav Physiol 2004; 34: 7981.

18. Thippeswamy $\mathrm{T}$, Morris $\mathrm{R}$. The roles of nitric oxide in dorsal root ganglion neurons. Ann N Y Acad Sci 2002; 962: 103110.

19. Aoki E, Semba R, Kashiwamata S. Evidence for the presence of L-arginine in the glial components of the peripheral nervous system. Brain Res 1991; 559: 159-162.

20. Ivaska J, Pallari HM, Nevo J, Eriksson JE. Novel functions of vimentin in cell adhesion, migration, and signaling. Exp Cell Res 2007; 313: 2050-2062. 